\title{
Dendritic nanostructures of silver: Facile synthesis, structural characterizations, and sensing applications
}

\author{
Xiaogang Wen, Yu-Tao Xie, Martin Wing Cheung Mak, Kwan Yee Cheung, Xiao-Yuan Li, \\ Reinhard Renneberg, Shihe Yang* \\ Department of Chemistry \\ The Hong Kong University of Science and Technology \\ Clear Water Bay, Kowloon, Hong Kong, China
}

\section{Supporting Information}

Figure S1 HRTEM image of a Ag nanodendrite (from Fig. 3C) in the joint region of a stem and a branch. The HRTEM image shows that the fringes of the nanodendrite in the stem/branch joint are not continuous.

Figure S2 Current signal vs. glucose concentration from raw data of Ag nanodendrite biosensors prepared with sol-gel solutions. These curves were used for constructing the calibration curves in Figure 7. Curve A: $60 \mu \mathrm{g}$; Curve B: $30 \mu \mathrm{g}$; Curve C: blank sample without Ag nanodendrites.

Figure S3 Cyclic voltammograms of a Pt electrode modified with Ag nanodendrites (red curve) with an anodic peak current of $+350 \mathrm{mV}$ and the corresponding bare $\mathrm{Pt}$ electrode (blue curve). Electrolyte: 0.05 M phosphate buffer saline (PBS). Scan rate: $200 \mathrm{mV} / \mathrm{s}$. 


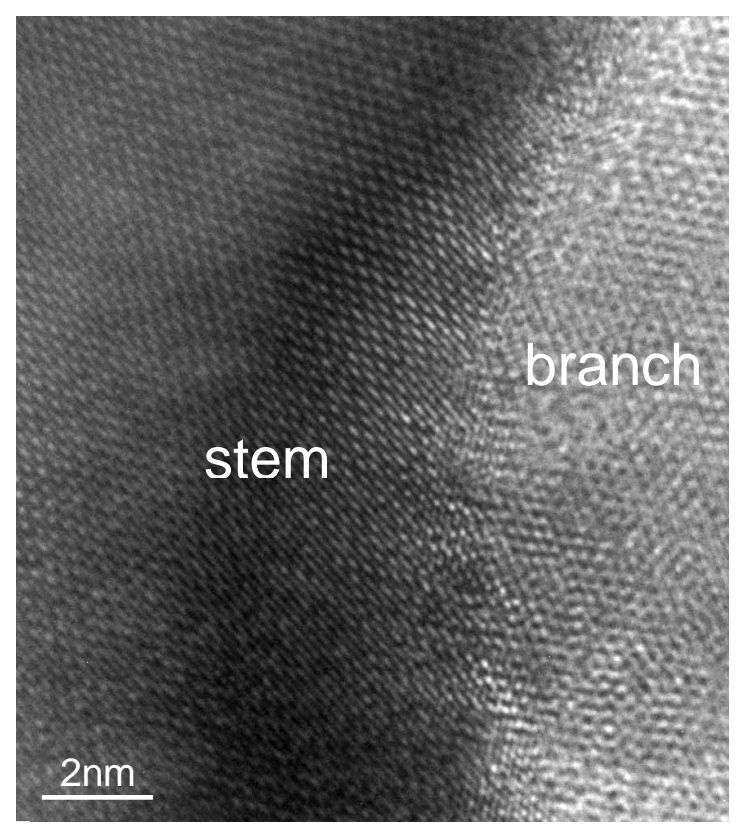

Figure S1 HRTEM image of a Ag nanodendrite (from Fig. 3C) in the joint region of a stem and a branch. The HRTEM image shows that the fringes of the nanodendrite in the stem/branch joint are not continuous. 


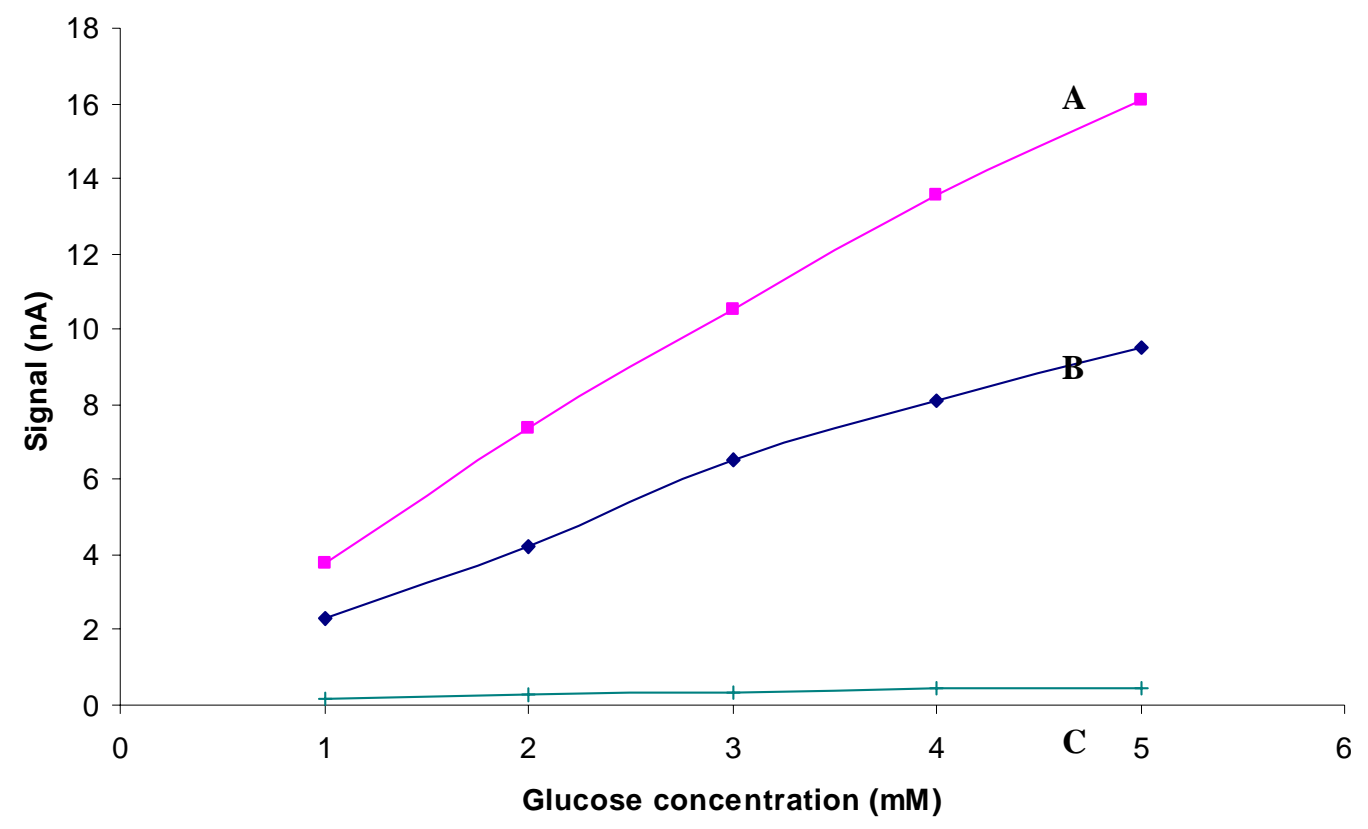

Figure S2 Current signal vs. glucose concentration from raw data of Ag nanodendrite biosensors prepared with sol-gel solutions. These curves were used for constructing the calibration curves in Figure 7. Curve A: $60 \mu \mathrm{g}$; Curve B: $30 \mu \mathrm{g}$; Curve C: blank sample without Ag nanodendrites. 


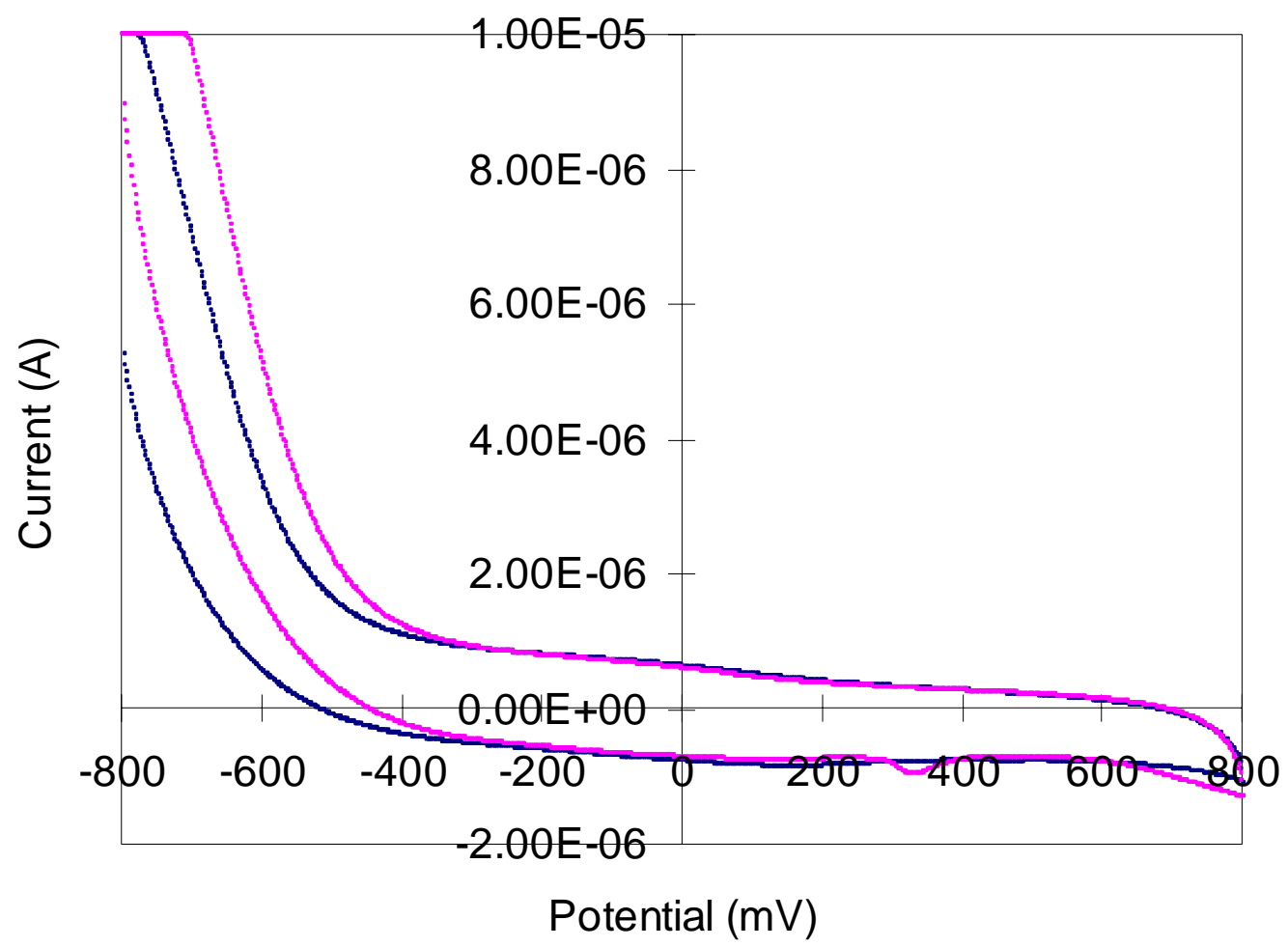

Figure S3 Cyclic voltammograms of a Pt electrode modified with Ag nanodendrites (red curve) with an anodic peak current of $+350 \mathrm{mV}$ and the corresponding bare $\mathrm{Pt}$ electrode (blue curve). Electrolyte: $0.05 \mathrm{M}$ phosphate buffer saline (PBS). Scan rate: $200 \mathrm{mV} / \mathrm{s}$. 